\title{
Visualizing the Macromolecular Organization of Chloroplast Membranes Using Cryo-Electron Tomography
}

D. Nicastro, ${ }^{*}$ J. Austin II, ${ }^{* *}$ J. Pierson, ${ }^{*}$ R. Gaudette, ${ }^{*}$ C. Schwartz, ${ }^{*}$ M. Ladinsky, ${ }^{*}$ L. A. Staehelin, ${ }^{* *}$ and J. R. McIntosh*

* Boulder Laboratory for Three-Dimensional Electron Microscopy of Cells, University of Colorado, Boulder, Colorado 80309-0347

** Department of Molecular, Cellular, and Developmental Biology, University of Colorado, Boulder, Colorado 80309-0347

The efficient harvesting of solar energy and its conversion into chemical potential energy are of great importance to all organisms. In chloroplasts the stacked photosynthetic membranes, or thylakoids, are a good model system for studying the molecular organization of photosynthesis. Thylakoids have been imaged in situ and their different functional states can be discriminated spectroscopically. Thylakoids can be purified for biochemical study, and there are hundreds of mutants with altered thylakoid membrane proteins, lipids, developmental patterns and structure. More recently, high-resolution crystal structures of all the major proteins found in the photosynthetic membranes have been determined [for review see 1,2]. For crystallographic studies, however, proteins are isolated from their cellular context and often crystallized under non-physiological conditions. Thus, despite the wealth of structural information available on individual proteins, much remains to be learned about protein conformation and organization under native conditions within the thylakoid membranes.

Freeze fracture studies in the 80's revealed the overall organization of protein complexes within the native thylakoid membrane [e.g. 3] and recent atomic force microscopic studies have provided additional insights into the architecture of native photosynthetic membranes from bacteria [2,4]. The latter imaging technique can reach a remarkable lateral resolution, but faces two major limitations: it provides information only about surface topographies, and when applied to biological tissue, the elasticity of the sample reduces the achievable resolution. We are therefore studying the threedimensional (3-D) structure of frozen-hydrated photosynthetic membranes using electron tomography (ET). ET is based on multiple views of a 3-D object obtained as it is tilted around an axis perpendicular to the electron beam. The resulting 2-D projection images of the object are recorded and used in weighted backprojections to reconstruct the original 3D volume.

A Tecnai F30 TEM (FEI, Eindhoven) equipped with an energy filter (GIF 2000, GATAN) was used to acquire several tilt series from each of several different preparations, including intact isolated and then cryo-sectioned spinach chloroplasts, isolated and plunge-frozen thylakoid membranes and intact Chlamydomonas cells. Using cryo-ET, we can compare the macromolecular organization of frozenhydrated material with the earlier freeze fracture studies, and there is the prospect of improving the image resolution to investigate how the component macromolecules interact with each other as neighbors both within and between thylakoid membranes. Our long range goal is to determine how the crystal structures of these complexes fit into the thylakoid membrane.

Preliminary results enable us to visualize the organization of the thylakoid stacks in reconstructions of frozen-hydrated sections of chloroplasts (Fig. A). In preparations of isolated thylakoids treated with digitonin to enrich for stacked membrane regions we have been able to study the fine structure of both isolated vesicles and stacked membranes (Fig. B-F). Our results confirm that thylakoid 
membranes are divided into specialized domains which differ in their macromolecular composition. So far we have been able to identify ATP synthase particles on the surface of the stroma thylakoids (Fig. A,B,F-H) and oxygen-evolving-complexes that clearly protrude above the membrane (Fig. BE). We have also found paracrystalline arrangements of unidentified macromolecular complexes, similar to those seen in freeze fracture images. To improve the signal-to-noise ratio in the tomographic reconstructions and thus to enhance our resolution, we are now using image processing tool such as correlation averaging (Fig. G,H). These techniques should enable us to better understand the macromolecular organization of the photosynthetic machinery in situ at a new level of structural detail.

\section{References}

[1] L.A. Staehelin, Photosynth Res. 76 (2003) 185-196. [2] S. Bahatyrova et al., Nature 430 (2004) 1058-62. [3] L.A. Staehelin, Biochim Biophys Acta 408 (1975) 1-11. [4] S. Scheuring et al., Proc Natl Acad Sci U S A. 101 (2004) 11293-7. [5] This work was supported by a NIH grant (RR00592) to JRM.

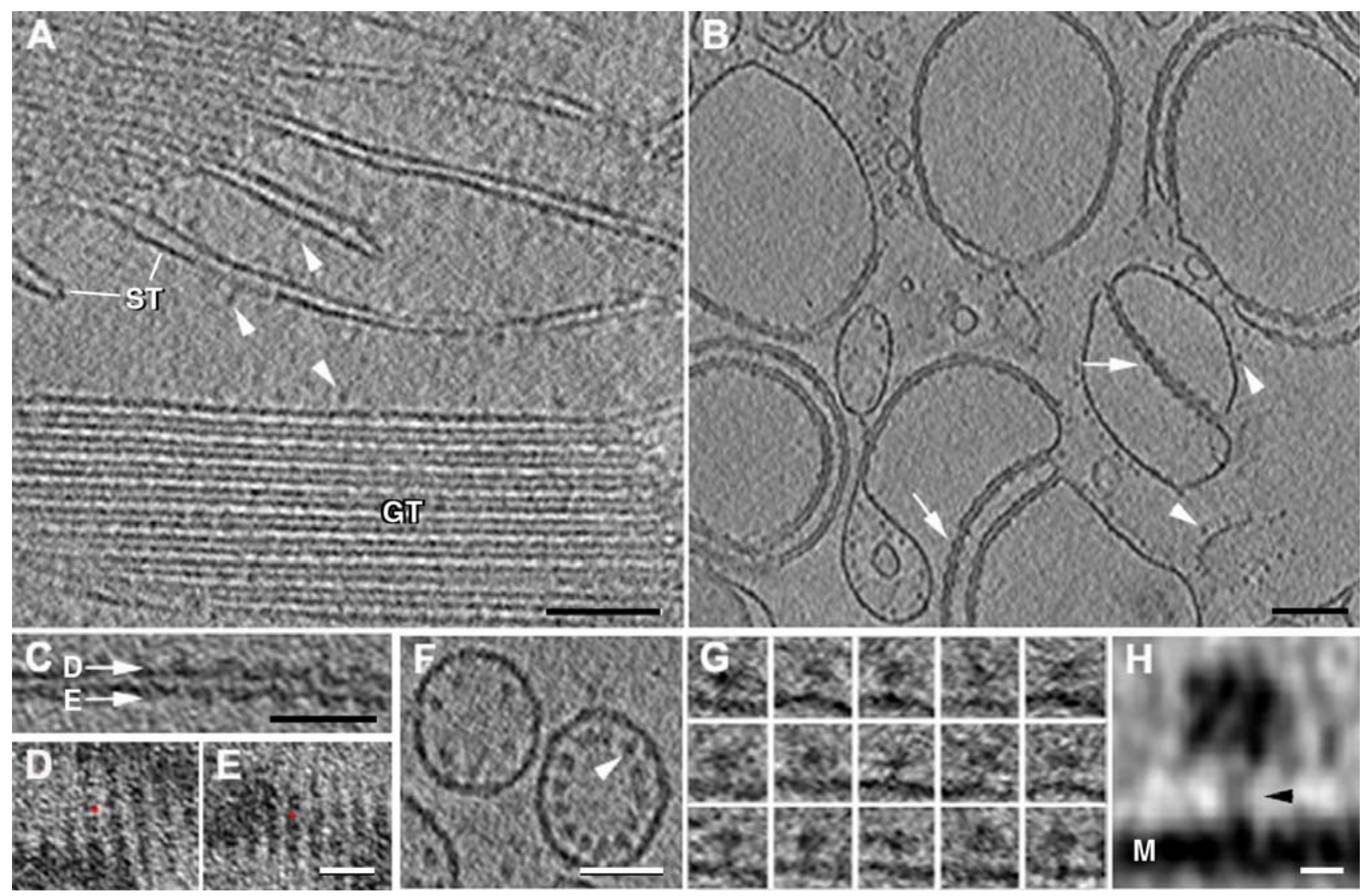

Fig. Macromolecular Organization of Chloroplast Membranes. A. $\sim 15 \mathrm{~nm}$ thick tomographic slice of a frozen-hydrated section of isolated spinach chloroplasts. Arrowheads, ATP synthase molecules; ST, stroma thylakoid; $G T$, grana thylakoid. B. $\sim 8 \mathrm{~nm}$ thick tomographic slice of isolated plunge-frozen thylakoids. Arrowheads, ATP synthases; arrows, oxygen evolving complexes. C. Detail of B showing oxygen evolving complexes. $D$ and $E$ indicate the planes shown in D and E, respectively. D,E. Top and bottom membrane from $\mathrm{C}$ rotated $90^{\circ}$ around the $\mathrm{x}$-axis; red dots indicate same position. F. Detail of B showing ATP synthase containing vesicles. Arrowhead, ATP synthase. G. Gallery of $\sim 3 \mathrm{~nm}$ thick tomographic slices displaying an ensemble of ATP synthase particles used to generate a 3-D average. H. Central slice of 3-D averaged ATP synthase generated from 35 particles. Scale bars: $100 \mathrm{~nm}$ (A,B); $50 \mathrm{~nm}(\mathrm{C}-\mathrm{F}) ; 5 \mathrm{~nm}(\mathrm{H})$. 